\title{
Development of Wearable Micro-Actuator Array for 3-D Virtual Tactile Displays
}

\author{
Zoltan Szabo, Eniko T. Enikov*
}

University of Arizona, Tucson, USA.

Email: *enikov@email.arizona.edu

Received April 14 $4^{\text {th }}, 2012$; revised May 15 $5^{\text {th }}, 2012$; accepted May 25 ${ }^{\text {th }}, 2012$

\begin{abstract}
A novel 4 by 4 array of electromagnetic micro-actuators operating on the principle of voice-coil actuators is presented. The intended application of the array is dynamic tactile stimulation, where multiple actuators generate an illusion of touching a moving pattern. In comparison to earlier designs [1-3], the device has smaller dimensions of $2.28 \mathrm{~mm}$ in diameter and $7 \mathrm{~mm}$ in length, which allowed its use in an array capable of hosting up to a 5 by 5 set of actuators with a rectangular shape covering an area of $18 \mathrm{~mm}$ by $21 \mathrm{~mm}$. Using finite element analysis of several conceptual designs of actuators $[1,4,5]$, it was established that the voice-coil type device (where the coil is the moving part) has most beneficial characteristics for the envisioned application. These include sufficient force over a relatively large distance, allowing tactile stimulation of surfaces with irregular shape, fast response, and small foot-print that matches the density of the tactile sensory neurons in the human finger. Eexperimental evaluation of the operation of neighboring actuators spaced at $3.3 \mathrm{~mm}$ apart, indicates that there is no crosstalk between the actuators. The resulting density exceeds that of previously reported alternative designs based on moveable permanent magnets $[4,6]$. Static force measurement indicate that each micro-actuator can produce at least $26 \mathrm{mN}$ of repulsive force over a stroke of $2100 \mu \mathrm{m}$ with a peak force of $34 \mathrm{mN}$. The driving circuit operates at $13.5 \mathrm{~V}$ and generates a vibration frequency of up to $265 \mathrm{~Hz}$ without significant change of the force-displacement characteristics. In the higher frequency range (above $100 \mathrm{~Hz}$ ) the actuator provides at least 15 $\mathrm{mN}$ of force over a slightly reduced stroke of $2300 \mu \mathrm{m}$, and a peak force of $21 \mathrm{mN}$. All of the above parameters meet the required threshold values of tactile human perception known from $[2,3]$.
\end{abstract}

Keywords: Micro-Actuator; Voice-Coil Microactuator; Tactile Display

\section{Introduction}

Communication devices for visually impaired people are generally limited to transduction of written text into a continuously perceived and spatially discriminated format or a synthetic language such as Braille. With the emergence of the World-Wide-Web (WWW), a significant interest has arisen in developing graphical displays for visually impaired individuals [7]. Recently, several academic and commercial organizations have put forward opto-electronic devices aimed at the capture and display of text and graphics in real-time using a twodimensional (2D) tactile map. While this represents an improvement of the traditional Braille-only language, a 2D projection of our three-dimensional (3D) world often remains difficult to interpret.

A new direction of development of three-dimensional display of shapes is represented by a novel virtual tactile display pioneered by one of the authors [7]. The shape perception is based on the interaction between the human

"Corresponding author. operator and a computer-generated virtual object. The reference shape is recorded through its coordinates for comparison. When the operator's finger is at the positions of the reference shape, the actuator is engaged and accordingly creates vibratory stimulation to human finger. At the positions out of the reference shape, the actuator remains off. The iteration of such tactile feedback loop produces and updates an estimated image of the reference shape in user's mind by one's continuous scanning and perceiving the boundary where the tactile stimulation occurs. Initial tests of the virtual 3D display indicated that efficient perception of corners and edges of object requires multiple actuators arranged into an array.

Over the past few years many actuator arrays have been reported for use in static tactile display (representing a fix physical tactile map), however use of such arrays in real-time virtual tactile display remains elusive. Examples of earlier efforts to produce a 3D tactile display include mechanical reproduction of a shape [5], the use of dynamically reconfigurable taxels (tactile pixels) based on piezoelectric biomorph reed cantilevers [8], use 
of passive [9], or motor-driven mechanisms [10,11]. More recent efforts include a pneumatic device [12]. However, none of these devices are mobile or small enough to be wearable. A separate body of research addresses the group of wearable tactile actuators. Most of these are based on the electromagnetic working principles. Review of previously reported devices shows that they produce relatively small forces $14 \mathrm{mN}$ [13], $6 \mathrm{mN}$ [14], $0.9 \mathrm{mN}$ [15], as a result of a trade-off between power and size of the actuator. More recent efforts to design smaller and more powerful actuators have led to devices based on moving permanent magnets with a diameter of 2 millimeters and a significant force peak ( 70.8 $\mathrm{mN}$ ), however the force-displacement characteristic is monotonously decreasing thus making it difficult to remain in contact with the curved surface of the finger. Furthermore, the output amplitude rapidly decreases at frequencies above $150 \mathrm{~Hz}$, which is the most effective range of tactile perception [16]. Another effort to construct a similar tactile array was an electromagnetic coildriven micro-actuator with a diameter of $3.8 \mathrm{~mm}$ and maximum force of $13 \mathrm{mN}$ [6]. The shortcomings of this design is the small force, and the large overall size of the array $(30 \times 30 \times 20 \mathrm{~mm})$, and the reported crosstalk between the actuators when at spacing distance below 5 $\mathrm{mm}$.

This article reports on the development and testing of a novel array based on miniature actuators. Section 2 includes a description of the required actuator performance for the envisioned application and an exploration of various actuator layouts aimed at identifying an optimal geometry. Section 3 presents the challenges of integrating the actuator into an array of closely spaced actuators, while Section 4 is devoted to the experimental analysis of the performance of the actuator array and validation of the finite element model used in the design of the actuator. Using the validated model, in Section 5 is has been shown that further miniaturization is possible while maintaining the minimal performance required for successful tactile stimulation.

\section{Design of a Single Actuator}

\subsection{Requirements for an Actuator}

Most of the design requirements are derived from physiological studies of human tactile perception. These include:

- Use of mechanical excitation [17], as opposed to electrical, thermal, or ultrasound. Mechanical stimulation is generally regarded as safe as opposed to electrical and ultra-sound excitation.

- Ability to create a moving stimulus (not stationary, so operating at a vibrating mode) [18].

- Ability to generate fluctuating stimulus (variable magnitude and frequency of the force produced by the actuator on the fingertip) [18].

- A minimal force of $5-10 \mathrm{mN}$ in order to increase the probability of successful perception [19].

- A practical upper limit on the diameter of the actuator is $3 \mathrm{~mm}$.

- A minimal actuator displacement of $5 \mu \mathrm{m}$ [20]. Due to the non-uniform surface and so the changing distance between the moving fingertip and the actuator this stroke may need to be orders of magnitude larger.

- The device should be light in weight so it doesn't cause extra fatigue to the user.

- The actuator including the connecting cables should not limit the natural motion of the human subject.

- The operating frequency should be around $250 \mathrm{~Hz}$, since this is more perceivable at low amplitudes of fingertip penetration [18].

\subsection{Analysis of Actuator Concepts}

Review of most common actuation modes reported in literature indicates that the electromagnetic actuation is the best match for the envisioned application [8-20]. One of the reasons behind this is that the magnetic field energy density is higher than the electric field energy density at the same energy input [16]. The magnetic field can be used to produce mechanical, translational movement based on the Biot-Savart and Lorentz laws. Within this group of actuators, common layouts include solenoid type devices, which apply a fixed coil (solenoid) and a moving ferromagnetic core, and voice-coil devices, where either the coil or a permanent magnet is the moving part, while the other one is fixed.

In order to decide which type of electromagnetic actuator to use, static-electromagnetic finite element analysis was carried out using a finite element code (ANSYS). The examined concepts are presented in Figure 1, were two of the solenoid devices, have a plunger as the moving part (Figure 1(a)), and two of the voice-coil types have the coil as the moving part (Figure 1(b)).

For comparative purposes, all concepts utilized the same outer diameter, current, number of turns, and current density (current times number of turns over the cross section of the coil). Furthermore, all magnetic and ferromagnetic material properties were kept unchanged in all four cases. The analysis examined the static electromagnetic behavior of the actuator, focusing on the repulsive force acting on the coil as a function of the position of the moving part (plunger or coil). The electromagnetic forces were recorded over fixed and identical positions for all four cases. The relative values of the computed forces are summarized in Table 1. The results of the force calculation show that concept \#3 provides the highest force due to the most favorable distribution of the 
magnetic field lines. For this reason, concept \#3 was chosen for further examination

\subsection{Dimension Examination Based on Finite Element Analysis}

In order to reduce the friction between the moving coil and its core, an ultra-thin walled $(32 \mu \mathrm{m})$ polyimide tube was used as a mandrel for producing the coil. The inner surface of the plastic tube is smooth enough to provide minimum friction, while the polyimide is stiff enough to provide a rigid base for the winding procedure. To focus the force in a smaller contact area to the fingertip, a plastic cap was added on the top of the coil. The schematic figure of the extended actuator is shown in Figure 2.

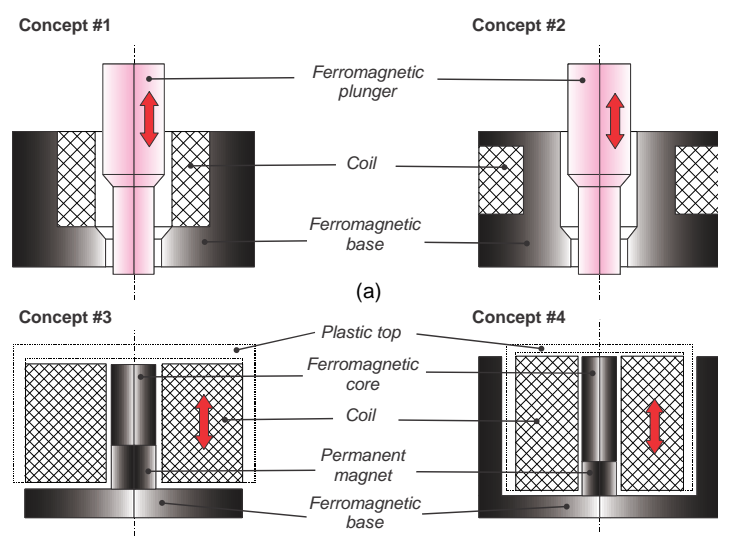

(b)

Figure 1. Examined concepts of electromagnetic actuators.

Table 1. Comparison of forces at different electromagnetic actuator concepts.

\begin{tabular}{cc}
\hline Concept \# & Force relative to concept \#1 \\
\hline 1 & $100 \%$ \\
2 & $42.8 \%$ \\
3 & $381.3 \%$ \\
4 & $324.9 \%$ \\
\hline
\end{tabular}

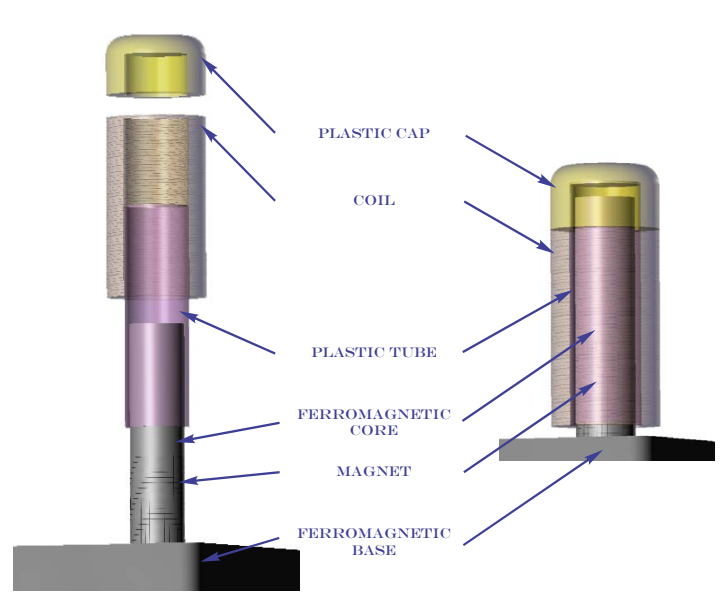

Figure 2. Schematic figure of the actuator.
A parametric optimization was then carried out in order to arrive at an optimal design maximizing the force.

A summary of the physical dimensions and material properties is shown in Table 2.

The parameters of the FE analysis are as shown in Figure 3.

Table 2. Technical data of the raw materials.

\begin{tabular}{lc}
\hline Wire & \\
\hline AWG & 43 \\
Magnet & $1.5875 \mathrm{~mm}$ \\
Diameter & $2.38125 \mathrm{~mm}$ \\
Length & $1.41 \mathrm{~T}$ \\
Residual flux density (Br max) & $883309.9 \mathrm{~A} / \mathrm{m}$ \\
Coercive force (H) & \\
Ferromagnetic core and base BH curve & $\mathrm{H}$ [A/m] \\
B [T] & 61.83 \\
0.8 & 66.92 \\
0.9 & 73.13 \\
1.0 & 222.81 \\
1.5 & 10583.80 \\
1.9 & \\
Plastic tube (polyimide) & $1.829 \mathrm{~mm}$ \\
Inner diameter & $1.893 \mathrm{~mm}$ \\
Outside diameter & \\
\hline
\end{tabular}

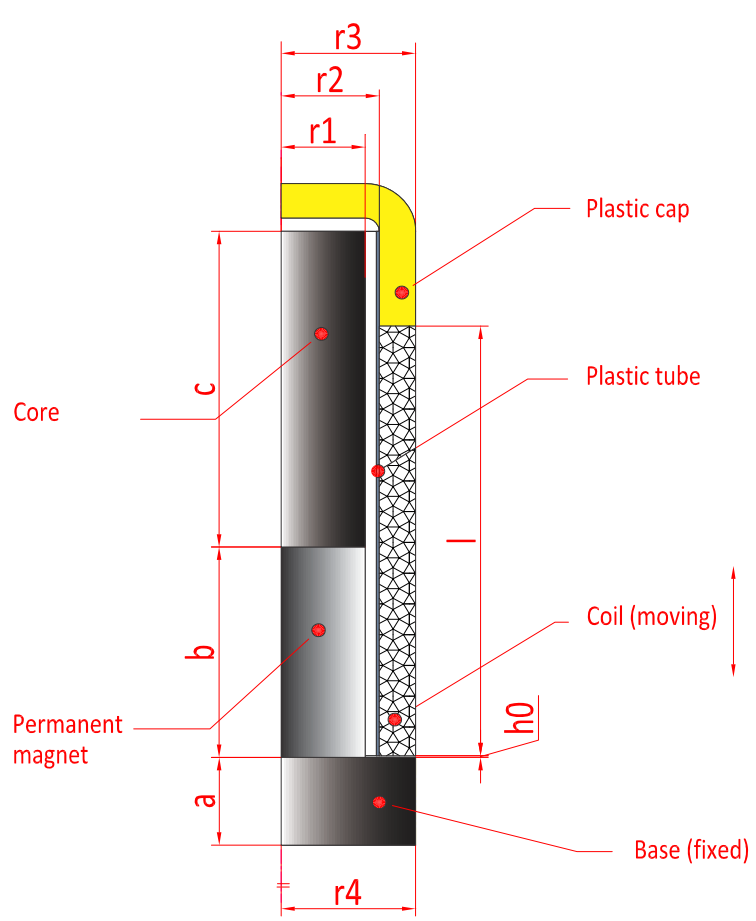

Figure 3. Parametric figure of the actuator. 
PLANE53 element was used in the static electromagnetic analysis to examine the force at different positions of the coil by changing the value of $h 0$ in increments of $300 \mu \mathrm{m}$. At each step the model was re-meshed and the force was recalculated by virtual work theory using MAGSOLV and FMAGSUM macros. During the dimension analysis, the displacement expressed with $h 0$, the coil inner and outer radii $r 2$, and $r 3$, respectively, and the core radius $r 1$ were kept constant (See Figure 3). By iteratively changing the remaining geometric parameters until the maximum force of $30.12 \mathrm{mN}$ is obtained with $200 \mathrm{~mA}$ of current. Sensitivity analysis of the predicted force showed that

- $r 4$ - can be changed in the range of 1.15 and $1.45 \mathrm{~mm}$ without affecting the achieved force.

- $a$-can be changed between 0.5 and $2 \mathrm{~mm}$ without changing the achieved force.

- The optimal ratio of $b$ and $c$ should be $2: 3$. Since the length of the magnet is given, the optimal length of the coil, $l$, can be determined.

The corresponding optimal parameter values are shown in Table 3.

\section{Design of 4 by 4 Array}

\subsection{Requirements for an Array}

The design of a miniature wearable tactile display involved technological and physiological constraints as listed below.

- Accurate representation of characters and shapes require at least 4 by 4 , preferably 4 by 5 or 5 by 5 set of actuators in a row-column pattern.

- Neither physical nor electromagnetic crosstalk between the electromagnetic actuators.

- For an array of actuators, the inter-actuator distance should not be smaller than $2 \mathrm{~mm}$, since this is the average spatial acuity of the tactile perception of young and middle age human's finger [2], also known as the two point threshold or two-point discrimination distance [19]. Since the practical upper limit on the diameter of the actuator is $3 \mathrm{~mm}$, for a 5 by 5 array the spacing between the actuators should be not less than $3 \mathrm{~mm}$.

- The overall size of the actuator should match the size of the average fingertip $(12 \times 15 \mathrm{~mm})$.

- The device should be light in weight so it doesn't cause extra fatigue to the user, but at the same time it must be robust enough to avoid easily being destroyed by the consumer.

- The array including the connecting cables should not limit the natural motion of the human subject.

- The array must provide convenient way to guide the wires of the coils.

- Each actuator should be individually controllable through the driving circuit.
- Providing potential to connect the array to mobile devices.

\subsection{Design of the Array}

The array is designed to have two sets of components. The bottom unit includes the ferromagnetic base with the permanent magnets and the ferromagnetic cores attached to it. Since the bottom unit is made of two different materials that must interact magnetically, the connection of the components is resolved by using strong adhesive. In order to provide the opportunity to expand the array of coils from 4 by 4 , the bottom unit consists of 25 cores providing hosting capability to a 5 by 5 array of coils. The bottom of the magnets is sitting $0.4 \mathrm{~mm}$ deep underneath the top surface of the base. This indentation provides guidance to position the magnets perpendicularly to the surface of the base. To assist alignment during the assembly, the ferromagnetic cores and magnets were placed in tubular guides that allowed the adhesive joints to set despite magnetic forces between the permanent magnets.

Even though the base is capable of serving 25 coils, for the first attempt, shown on Figure 4, the top unit was made as a 4 by 4 set of coils with the structure described above in the article.

In order to provide guidance for the pairs of wires originating from the coils, the base contains small holes through which the wires are free to move. The wires of the coils are pulled through the wire guide holes, this way they don't interfere with each other which is an essential need due to the delicateness of the $0.0558 \mathrm{~mm}$ diameter wire.

There are 3 assembly holes providing platform for using screws to attach the array to other parts. The structure of the bottom unit is shown on Figure 5.

Table 3. Specifications of the actuator.

\begin{tabular}{cc}
\hline Parameter & Value \\
$r 1$ & $0.79375 \mathrm{~mm}$ \\
$r 2$ & $0.9465 \mathrm{~mm}$ \\
$r 3$ & $1.14 \mathrm{~mm}$ \\
$r 4$ & $1.14 \mathrm{~mm}$ \\
$a$ & $2 \mathrm{~mm}$ \\
$b$ & $2.38125 \mathrm{~mm}$ \\
$c$ & $3.6 \mathrm{~mm}$ \\
$l$ & $4.9 \mathrm{~mm}$ \\
N (number of wire turns) & 320 \\
Overall length (without cap) & $7 \mathrm{~mm}$ \\
Overall mass (core ${ }^{*}+$ coil) & $207.1 \mathrm{mg}(135.7+71.4 \mathrm{mg})$ \\
\hline
\end{tabular}

${ }^{*}$ Core, base and the permanent magnet. 


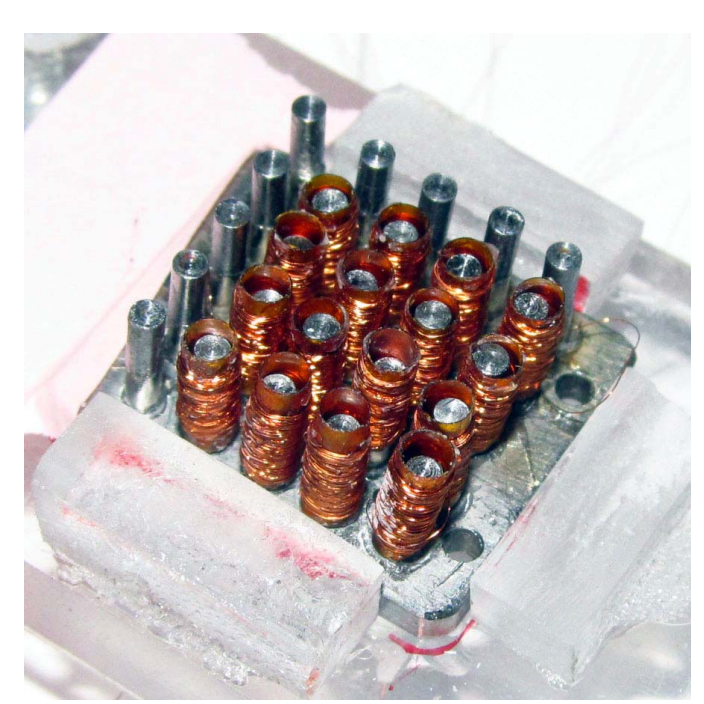

Figure 4. Photo of the array (bottom unit and coils).

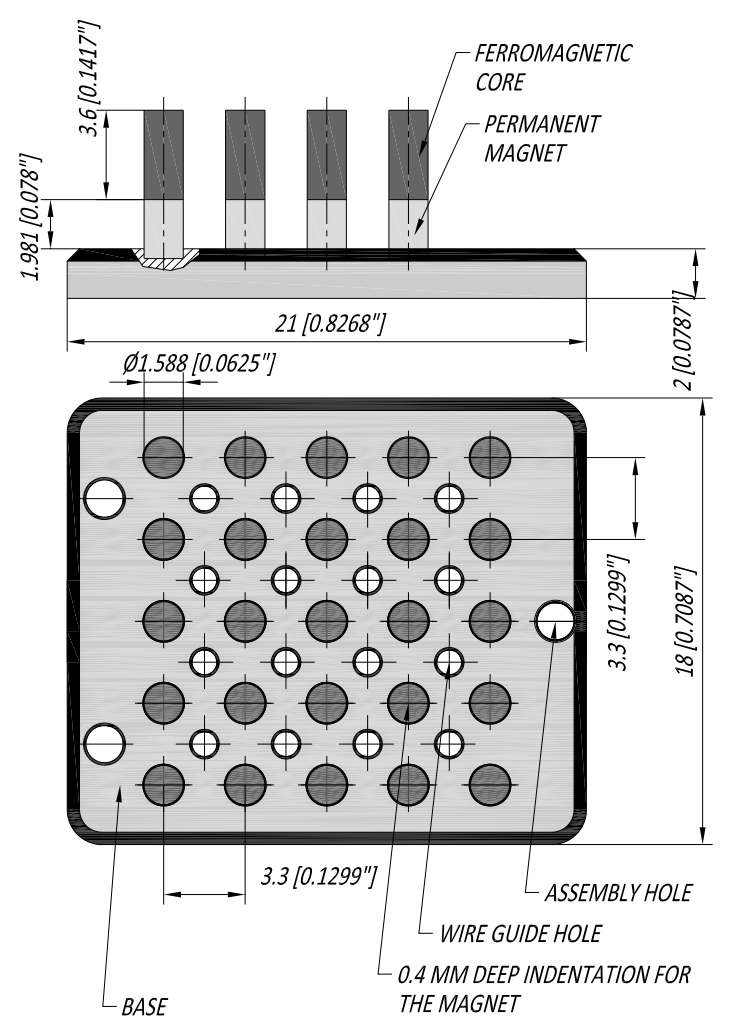

Figure 5. Structure of the bottom unit.

\subsection{The Driving Circuit for Analysis}

Both static and dynamic measurements were performed on an individual actuator. For the static measurements a constant DC power supply was needed that was directly connected to the coil. For the dynamic measurements a driving circuit that consists of a capacitor charged to the voltage of the power supply and released through the actuator's coil using field effect transistor (FET) switches was used. In order to predict the peak current in the circuit the transfer function of the RLC circuit was built, which is the following

$$
I(s)=\frac{C V_{0}}{L C s^{2}+R C s+1}
$$

where $C=96 \mu \mathrm{F}$ is the capacitance, $V_{0}=13.5 \mathrm{~V}$ is the DC power supply's voltage, $L=0.125 \mathrm{mH}$ the measured inductance of the coil, $R=17.7 \Omega$ the measured resistance of the coil. By taking the inverse Laplacetransform of the transfer function, the peak current of $i_{\max }=0.21$ A at $5 \mathrm{~ms}$ was established.

For possible resonance-phenomenon occurrence the damped natural frequency $\omega_{0}$ of the electrical system was calculated (2).

$$
\omega_{0}=\frac{1}{\sqrt{L C}}=1453 \mathrm{~Hz}
$$

The driving circuit of the array consists of 16 sets of the components of the single dynamic system, all controlled by a PIC16F690 microcontroller.

\section{Performance Analysis}

Performance analysis was carried out on individual actuators since the array is equipped with identical coils.

\subsection{Static Performance}

The FE analysis described in Section 2.3 lead to a forcecurrent and to a force-displacement characteristic of the actuator. Static force measurements were carried out using the setup shown in Figure 6. Briefly, it consists of a power supply, wiring to the actuator, the actuator, a micrometer mounted on a fixed column, and a scale.

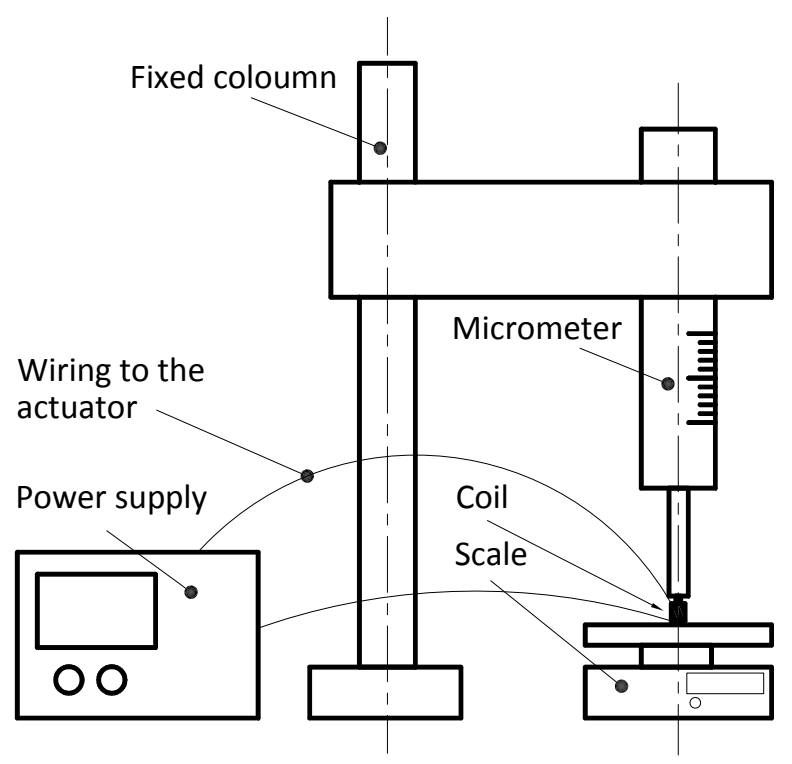

Figure 6. Measurement set up. 
Care was taken to make sure that the scale always remains at zero when the power is not supplied and that the coil is always perpendicular to the surface of the scale. When the power supply was turned on, the coil moved downwards, thus pushing on the scale.

For the force-displacement characteristics the DC voltage was kept constant, and before the change of each position of the actuator (adjusted by the micrometer and denoted by $h 0$ in the ANSYS simulation) the power supply was turned off, so the coil could cool down. The results of the ANSYS simulation and the measurements with the error bars are shown in Figure 7.

The force-displacement characteristic of the actuator is not monotonous. It increases until $1270 \mu \mathrm{m}$ with a peak of $29.4 \mathrm{mN}$ than it decreases. Nevertheless, the static measurement and simulation shows that the produced force is over $10 \mathrm{mN}$ until $4500 \mu \mathrm{m}$ stroke. A possible reason of the difference between the ANSYS and the measurement results is the non-ideal contact between the plastic tube and the core, which brings friction into the system. The other possible explanation is the heat generation in the coil that lowers the current, thus the force. Another reason why they might differ is the non-uniform distribution of the windings of the coils.

For the force-current characteristics the DC voltage was increased until the coil overheated and the position of the coil was adjusted to $1270 \mu \mathrm{m}$ which is the position of the force peak in Figure 7. The comparison of the FE simulation and real measurements with error bars are shown in Figure 8.

Figure 8 implies that there is a linearly proportional relationship between the applied DC current and the produced force, which is characteristic of Lorentzian forces. The curve does not go beyond $200 \mathrm{~mA}$, because the coil gets overheated at these excitation levels. The explanation of the difference between the results of two methods is most likely the heat generation that increases the resistance of the coil and it results a lower current. This is noticeable in the increasing errors as a function of increasing current (see error bars in Figure 8).

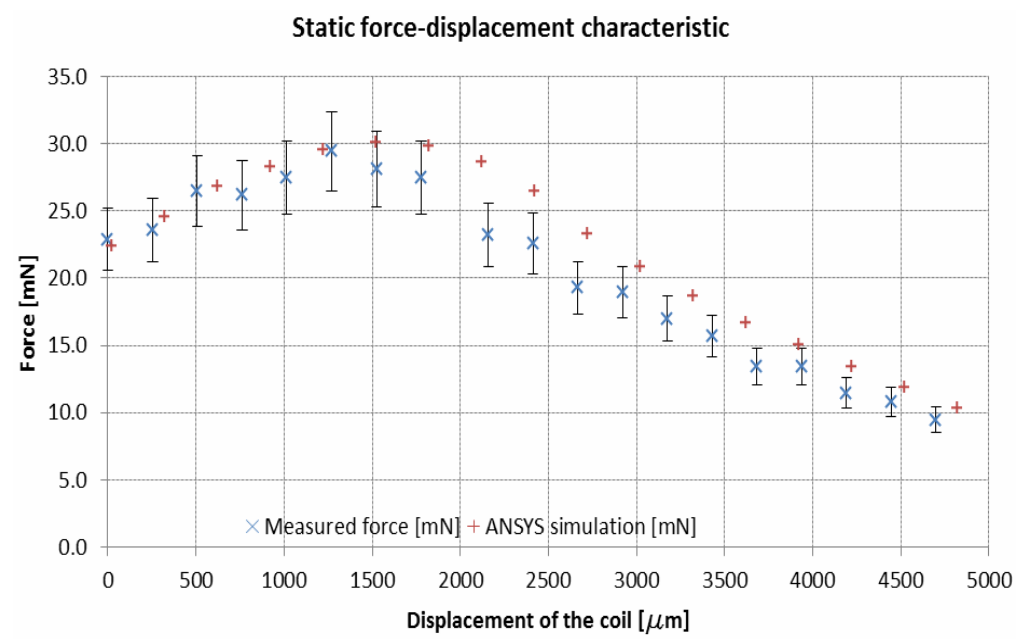

Figure 7. Static force-displacement characteristic.

Static force-current characteristic

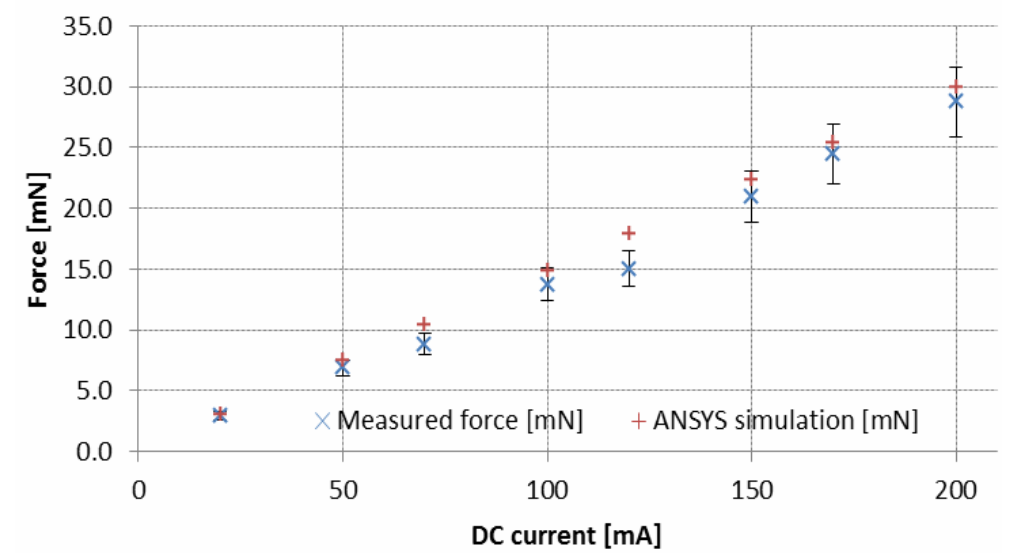

Figure 8. Static force-current characteristic. 


\subsection{Dynamic Performance}

The dynamic measurement involves the same measurement setup as described in Section 4.1 with the following extensions. A driving circuit (described in Section 3.3) between the coil and the power supply was used to power the coil intermittently. The driving circuit is controlled by a micro-processor connected to a PC via RS 232 link. The user can adjust the vibrating frequency with the computer.

After adjusting the capacitor charging voltage to 13.5 $\mathrm{V} D C$, a coil position is set by the micrometer, and then on the computer the vibration frequency is chosen. By reading the mass measured by the scale, one gets an averaged value of the achieved force since the scale is originally designed for static measurements. The dynamic force-displacement characteristics of the actuator at different frequencies are shown in Figure 9.

The higher frequencies resulted higher forces. This phenomenon is due to the larger testing frequencies being closer to the resonance frequency of the electrical circuit $(1453 \mathrm{~Hz})$, thus resulting higher current values which are linearly proportional to the force. In order to determine the possibility of mechanical resonance of the coils a single mass-spring vibrating model was built (Figure 10). The free end of the copper wire, presented as a spring, has the stiffness $k$, determined by the following equation

$$
k=\frac{d^{4} G}{8 D^{3} N_{a}}=1.42 \frac{\mathrm{N}}{\mathrm{m}}
$$

where $d=0.05588 \mathrm{~mm}$ is the diameter of the wire, $\mathrm{G}=$ 44.7 GPa the modulus of rigidity of copper, $D=2.3359$ $\mathrm{mm}$ the mean coil diameter and $N_{a}=3$ the number of active turns. The one degree of freedom undamped mechanical system has the natural frequency $\alpha$

$$
\alpha=\sqrt{\frac{k}{m}}=22.5 \mathrm{~Hz}
$$

where $m=71.4 \mathrm{mg}$ is the mass of the coil. Since the mechanical natural frequency is not close to the testing frequencies it is stated that the force differences at different frequencies is not the result of the properties of the mechanical oscillating system.

The difference between the forces decreases by the end of the displacement axis which is the result of the limited B field provided by the fixed permanent magnet. It is well observable that the force peak is about at the same position as in the static case. Some of the measurement points in $265 \mathrm{~Hz}$ and $205 \mathrm{~Hz}$ show peaks that doesn't fit the rest of the curve. These points are due to the resonance of the scale.

Comparing the dynamic and the static force-displacement characteristics it can be stated that the dynamic measurements resulted lower forces. Excluding the resonance points, the highest dynamic force peak was 21.58 $\mathrm{mN}$ at $1270 \mu \mathrm{m}$ while the static peak was $29.4 \mathrm{mN}$. Such a difference might be the result of the averaging method of the scale. Another explanation is that the current doesn't reach the peak calculated in Section 3.3 (due to the heat generation) so the force cannot grow high enough either.

\section{Dynamic force-displacement characteristic}

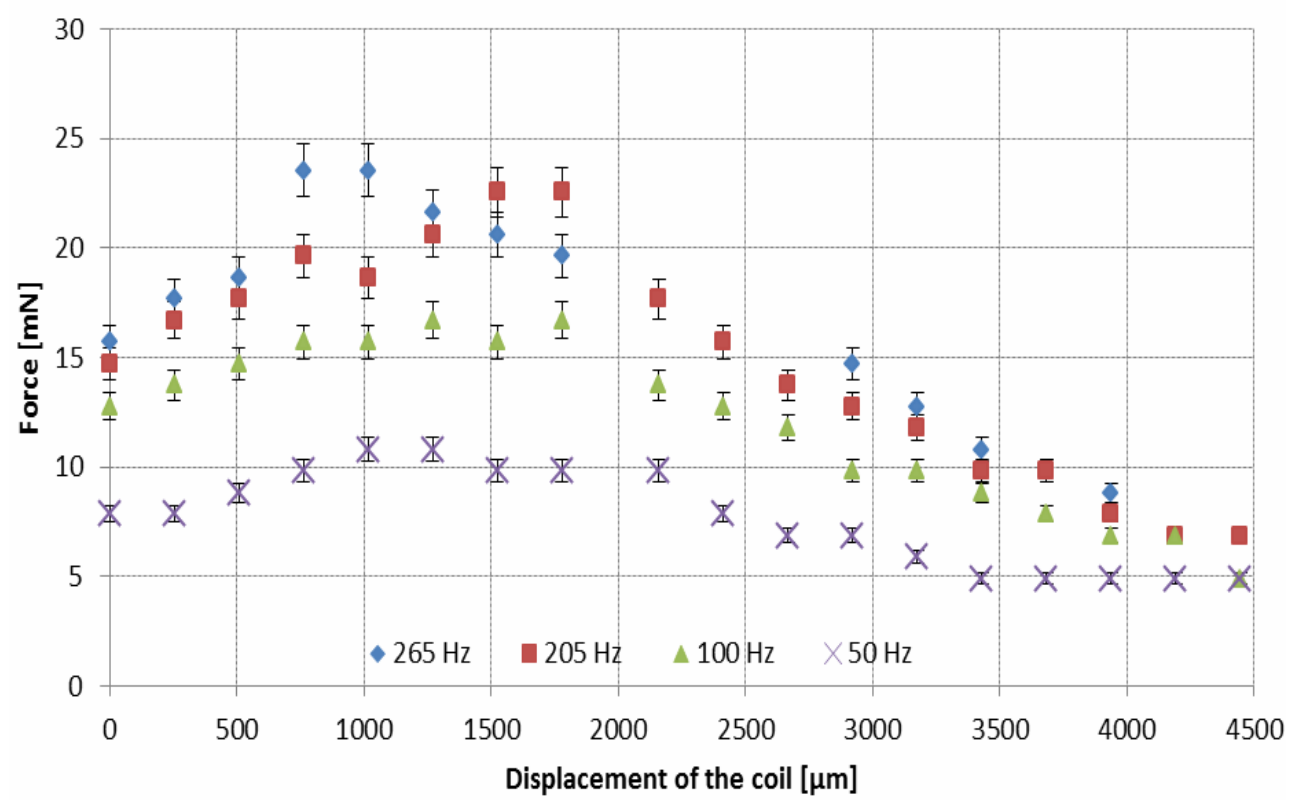

Figure 9. Dynamic force-displacement characteristic. 


\subsection{Tactile Perception Analysis}

While human subject tests are not yet available, comparison with previous devices [7] indicates that in a dynamic mode, the excitation on the fingertip is perceivable in all the described frequencies all over the presented displacement.

It is known from the literature [18] that the moving stimulus (vibro-tactile) is more perceivable than static however they have the same magnitude of force. This explains why the $5-10 \mathrm{mN}$ range remains perceivable in vibration mode. The satisfyingly working coils were placed into an array for generating shapes recognizable to human beings. The previously presented ASSEMBLY coded microcontroller drives the coils, shaping different characters such as letter $\mathrm{Z}, \mathrm{O}, \mathrm{N}, \mathrm{X}, \mathrm{L}$. This gives a future opportunity to test human subjects on perceiving

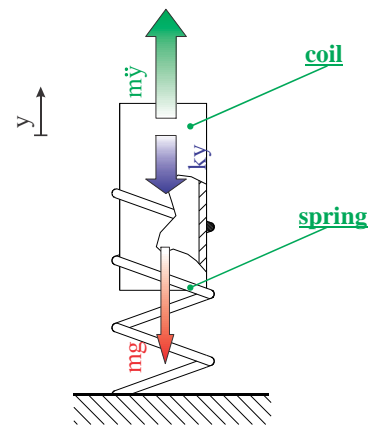

Figure 10. The mechanical oscillating model. characters and shapes with the use of this device. Figure 11 shows the illustration of activated coils presenting letter Z.

\section{Miniaturization to Threshold Limit}

A further analysis was carried out to explore the possibility of the miniaturization of the device. Since the reduction of the inter-coil distance, through the reduction of the diametric dimensions, leads to unwanted crosstalk between the coils, the diametric dimensions are kept at the already existing actuator's values (Table 3 ). In the miniaturization analysis the length-wise dimensions got reduced. The scaled illustrations of the miniature and the existing actuators and the magnetic field lines, generated by static-electromagnetic numerical analysis, are presented in Figure 12.
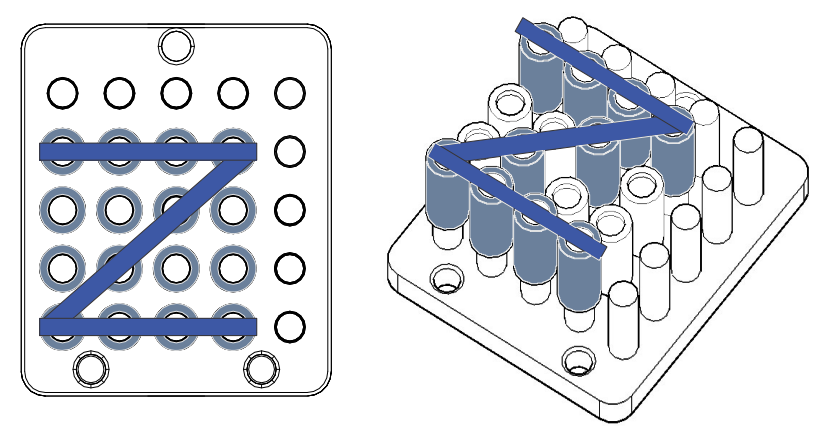

Figure 11. Coils forming the letter $\mathrm{Z}$.

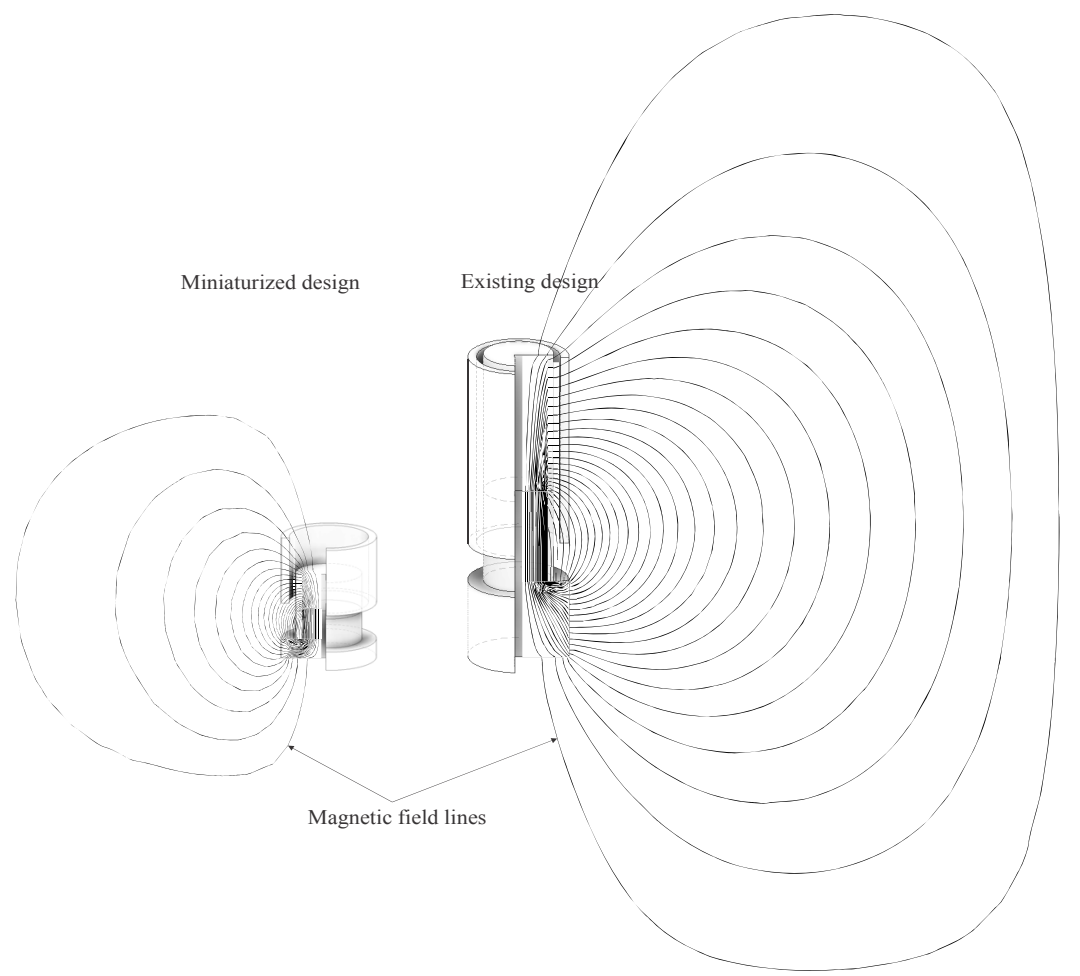

Figure 12. Scaled drawings of the existing and miniature designs with magnetic field lines. 
The reduced overall height makes the actuators more applicable for use in thinner devices. As the finite element analysis points it out, the reduction of the height lowers the achievable forces. Since the threshold limit of tactile perception in human beings is $5-10 \mu \mathrm{N}$ force, the miniaturization has limitations in terms of minimum dimensions. To satisfy the stroke requirement, discussed in Section 2.1, the necessary maximum displacement where there is still perceivable force was chosen to be $1.5 \mathrm{~mm}$. With these expectations the optimal design was established and simulated with static, electromagnetic finite element analysis. The results of the force calculations are presented in Figure 13.

The comparison of the parameters, introduced in Figure 3 , in the miniature and the existing designs are shown in Figure 14.

\section{Static force-displacement characteristic}

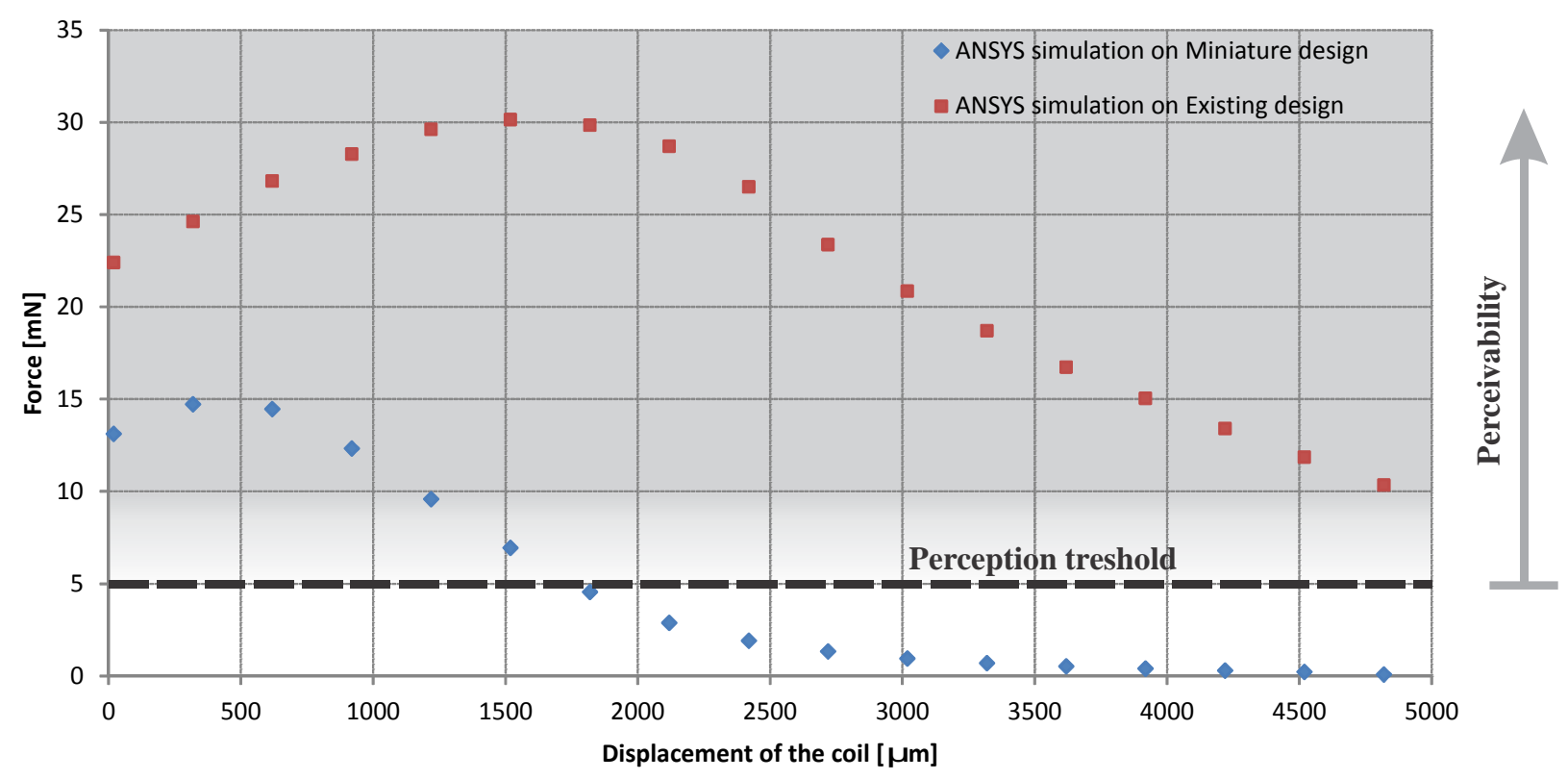

Figure 13. Comparison of static forces.

comparison of Miniature and Existing design in length-wise dimensions

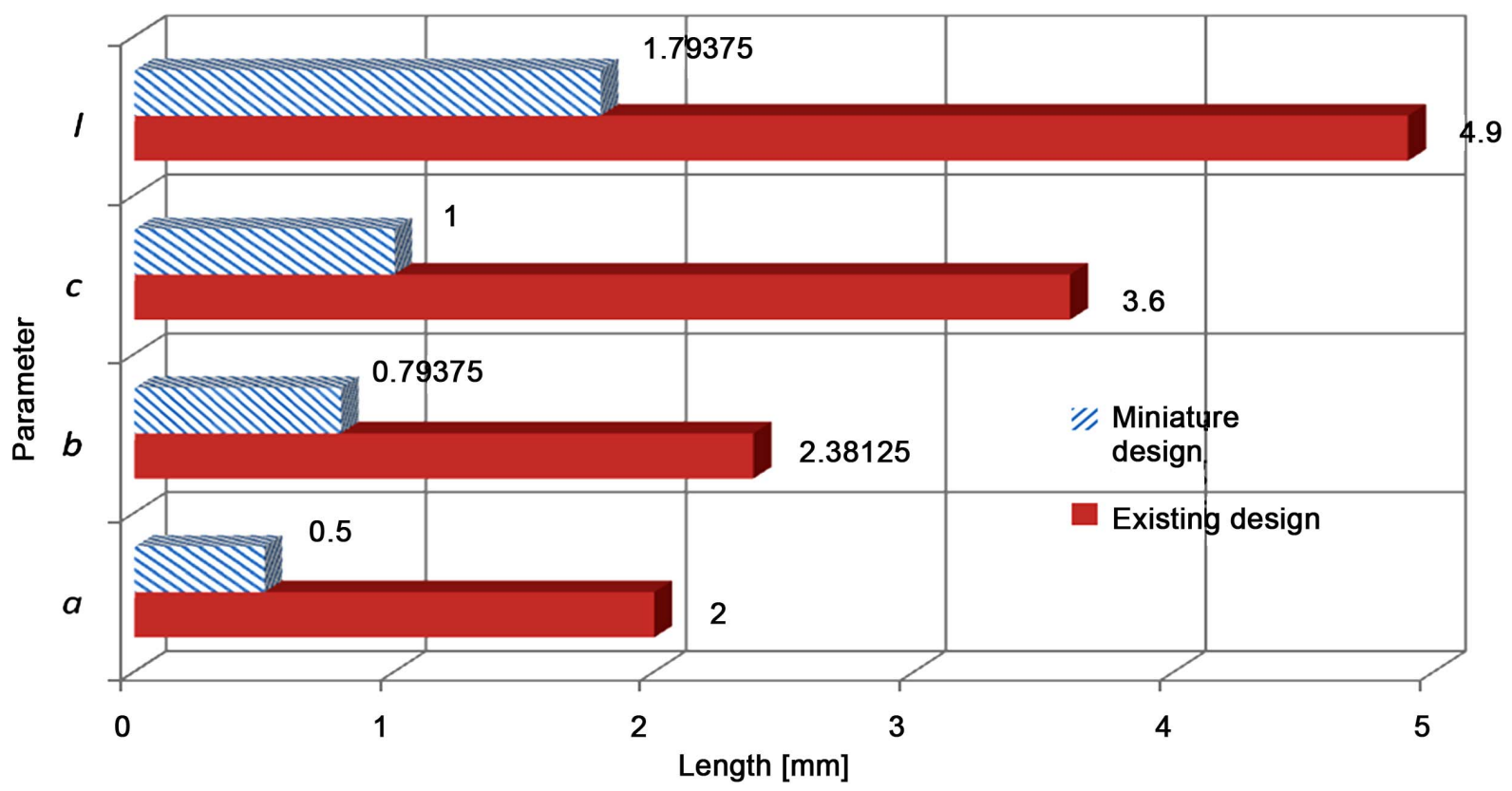

Figure 14. Comparison of parameters. 
As a consequence of the miniaturization, the volume of the coils in a 5 by 5 array reduces by $71 \%$ (the number of turns in each coil reduces to 93 from 320), while the volume of the base component of the array reduces by $75 \%$. The drop in volume is linearly proportional with the drop in weight. This weight reduction makes the ultimate design even more wearable for human beings.

\section{Conclusions}

A novel voice-coil micro-actuator and its extension to a 4 by 4 array are presented in this paper. The electromagnetic device satisfying all the requirements listed in Section 2.1 is suitable for use in virtual tactile displays, since it has a maximum diameter of $2.28 \mathrm{~mm}$, an overall length of $7 \mathrm{~mm}$, has a well perceivable force characteristic over a long stroke and it can be applied in a large frequency scale. The actuator is robust and capable of producing different letters that are recognizable by human touch.

Comparative analysis with earlier efforts based on movable permanent magnets of similar size indicate that the voice-coil actuators are more suitable for the envisioned application due to the increased stroke and elimination of cross-talk at small separations. Using a validated finite element model, it has been shown that micro voice-coil actuators can be further miniaturized reaching a size comparable with the two-point discrimination distance in human fingers.

\section{Acknowledgements}

The authors acknowledge the support for this work by the U.S. Dept. of Education (\#P116J080016), the Atlantis program of the European Commission (2008-1767/001-001 CPT USMOBI), support from National Science Foundation Grant 0856761,0927661 , and by a grant from the Rosztoczy Foundation.

\section{REFERENCES}

[1] F. M. Vidal-Verdu, "Graphical Tactile Displays for Visually-Impaired People," IEEE Transactions on Neural Systems and Rehabilitation Engineering, Vol. 15, No. 1, 2007, pp. 119-130. doi:10.1109/TNSRE.2007.891375

[2] J. C. Stevens, "Aging and Spatial Acuity of Touch," Journal of Gerontology: Psychological Sciences, Vol. 47, No. 1, 1992, pp. 35-40.

[3] K. B. Shimoga, "A Survey of Perceptual Feedback Issues in Dexterous Telemanipulation: Part II. Finger Touch Feedback," Virtual Reality Annual International Symposium, Seattle, 18-22 September 1993, pp. 271-279. doi:10.1109/VRAIS.1993.380769

[4] K. Deng and E. T. Enikov, "Design and Development of a Pulsed Electromagnetic Micro-Actuator for 3D Virtual Tactile Displays," Mechatronics, Vol. 20, No. 4, 2010, pp. 503-509. doi:10.1016/j.mechatronics.2010.04.011

[5] M. Shimojo, et al., "Human Shape Recognition Perform- ance for 3-D Tactile Display," IEEE Transactions on Systems, Man, and Cybernetics-Part A: Systems and Humans, Vol. 29, No. 6, 1999, pp. 637-645.

doi:10.1109/3468.798067

[6] M. Benali-Khoudja, M. Hafez and A. Kheddar, "VITAL: An Electromagnetic Integrated Tactile Display," Displays, Vol. 28, No. 3, 2007, pp. 133-144. doi:10.1016/j.displa.2007.04.013

[7] K. Deng, E. T. Enikov and H. Zhang, "Development of a Pulsed Electromagnetic Micro-Actuator for 3D Tactile Displays," IEEE/ASME International Conference on Advanced Intelligent Mechatronics, Zurich, 4-7 September 2007, pp. 1-5. doi:10.1109/AIM.2007.4412457

[8] J. C. Bliss, et al., "Optical-to-Tactile Image Conversion for the Blind," IEEE Transactions on Man-Machine Systems, Vol. 11, No. 1, 1970, pp. 58-65. doi:10.1109/TMMS.1970.299963

[9] C. C. Collins, "Tactile Television-Mechanical and Electrical Image Projection," IEEE Transactions on ManMachine Systems, Vol. 11, No. 1, 1970, pp. 65-72. doi:10.1109/TMMS.1970.299964

[10] H. Iwata, et al., "Project FEELEX: Adding Haptic Surface to Graphics," Proceedings of the 28th Annual Conference on Computer Graphics and Interactive Techniques, Los Angeles, 12-17 August 2001, pp. 469-476. doi: $10.1145 / 383259.383314$

[11] Y. Kawai and F. Tomita, "Interactive Tactile Display System-A Support System for the Visually Disabled to Recognize 3D Objects," Proceedings of International ACM Conference on Assistive Technologies, Vancouver, 1996, pp. 45-50.

[12] T. Iwamoto, M. Tatezono, H. Shinoda, "Non-Contact Method for Producing Tactile Sensation Using Airborne U1trasound," Proceeding EuroHaptics '08 Proceedings of the 6th International Conference on Haptics: Perception, Devices and Scenarios, Madrid, 10-13 June 2008, pp. 504-513. doi:10.1007/978-3-540-69057-3 64

[13] M. I. Koo, et al., "Development of Soft-Actuator-Based Wearable Tactile Display," IEEE Transactions on Robotics, Vol. 24, No. 3, 2008, pp. 549-559. doi:10.1109/TRO.2008.921561

[14] T. Fukuda, et al., "Micro Resonator Using Electromagnetic Actuator for Tactile Display," Proceedings of the International Symposium on Micromechatronics and $\mathrm{Hu}$ man Science, Nagoya, 5-8 October 1997, pp. 143-155. doi:10.1109/MHS.1997.768872

[15] H. K. Kim, et al., "Fabrication and Test of a Micro Electromagnetic Actuator," Sensors and Actuators A: Physical, Vol. 117, No. 1, 2005, pp. 8-16. doi:10.1016/j.sna.2003.10.079

[16] A. T. Kern, "Engineering Haptic Devices, A Beginner's Guide for Engineers," Springer-Verlag, Berlin, 2009.

[17] K. Deng, "Development of Virtual 3D Tactile Display Based on Electromagnetic Localization," Ph.D. Thesis, Graduate College the University of Arizona, Tucson, 2009.

[18] M. Critchley, "Tactile Thought, with Special Reference to the Blind," Proceedings of the Royal Society of Medicine, Vol. 46, No. 1, 1952, pp. 27-30. 
[19] A. Pascual-Leone and F. Torres, "Plasticity of the Sensorimotor Cortex Representation of the Reading Finger in Braille Readers," Brain, Vol. 116, No. 1, 1993, pp. 39-52. doi:10.1093/brain/116.1.39
[20] A. K. Kaczmarek, et al., "Electrotactile and Vibrotactile Displays for Sensory Substitution Systems," IEEE Transactions on Biomedical Engineering, Vol. 38, No. 1, 1991. 